\title{
Pituicytoma. Case report and literature review
}

Fernando Velandia Hurtado ${ }^{1}$, Jorge Enrique Aponte ${ }^{2}$, Humberto Madriñan ${ }^{3}$, Yency Forero ${ }^{4}$ and Yuliana Cuellar Anturi ${ }^{5}$

1. Universidad del Rosario, Escuela de medicina, grupo de Neurociencias (NEUROS), Bogotá Colombia

2. Internal Medicine, Hospital Universitario de la Samaritana, Hospital de Santa CLara. Bogotá Colombia

3. General Medicine physician, Universidad del Rosario, Bogotá Colombia

4. Internal Medicine, Hospital Universitario de la Samaritana, Colombia

5. General medicine physician, Universidad del Tolima, Colombia

\section{CASE STUDY}

Please cite this paper as: Velandia Hurtado F, Aponte JE, Madriñan $H$, Forero $Y$, Anturi YC. Pituicytoma. Case report and literature review. AMJ 2018;11(4):240-244. https://doi.org/10.21767/AMJ.2018.3390

\section{Corresponding Author:}

Jorge Enrique Aponte

Internal Medicine, Hospital de la Samaritana, Hospital de Santa clara Bogotá, Colombia

Email: jorapon3777@hotmail.com

\section{ABSTRACT}

Pituicytoma is a rare glial tumour located in the sellar and/or suprasellar region of the brain, it originates from pituicytes, and they were included in the World Health Organization (WHO) Classification of Central Nervous System Tumours in 2017. As mentioned above it originates from pituicytes cells in the posterior pituitary near the infundibulum. Histological characteristics permits classification and identification among other central nervous system tumours localized in the posterior pituitary or infundibulum. WHO classification describes them as grades such as grade I tumour which has a differentiated structure composed of spindle-shaped cells presumably derived from pituicytes. We report the case of a 37-year-old woman who developed a suprasellar tumour and that it was discovered following a past medical history of amenorrhoea and migraines. Once the lesion was detected in images, the patient rejected surgical treatment even when she developed galactorrhea and preferred medical treatment with cabergoline. However, two years later she developed visual defects due to increase in size by that time accepting surgical treatment. A review of the literature including most common clinical and radiological features of this entity as well as treatment protocols proposed are discussed.

\section{Key Words}

Pituicytoma, sellar tumours, suprasellar tumours, glial tumours

\section{Implications for Practice:}

\section{What is known about this subject?}

Pituicytomas are rare primary tumours of the adult neurohypohysis which can be confused with pituitary adenomas.

\section{What new information is offered in this case study?} Here we present some clinical features that might guide successful surgical management and histopathological diagnosis of this tumour.

3. What are the implications for research, policy, or practice?

Given the extreme rarity of this tumour, case reports constitute the highest level of available evidence, and help inform clinical practice.

\section{Background}

Pituicytoma is a glial neoplasia located in the sellar and/or suprasellar region of the brain and originates from pituicytes and it is characterized by special and indistinguishable histological features have a small degree of histopathological characteristics. Pituicytes cells are located in the posterior pituitary near the infundibulum. ${ }^{1-4}$ Tumours in the posterior pituitary are infrequent ${ }^{5}$ and the majority reported are secondary to metastatic lesions from another primary most of the time with seeding through vascular and glandular circulation. ${ }^{6}$ When tumours originate as primary, they have a more granular appearance. ${ }^{1}$ 
Tumours in the sellar and supersellar regions are more frequently pituitary adenomas, craniopharyngiomas, meningiomas, granular cell tumours, gangliogliomas, suprasellar germinomas, lipomas, hamartomas, teratomas, and pilocytic astrocytomas. However, when pituicytoma is suspected one most distinguish its unique histological features and differentiate among other neoplasias developed in this location as primary tumors. ${ }^{7}$ As we mentioned above the World Health Organization's classification of tumours in the central nervous system, describes pituicytomas as a WHO 2017 grade I tumours, which compromises the posterior pituitary and/or its stem composed of spindle-shaped cells presumably derived from pituicyte cells. ${ }^{1,5,9}$ We present the case of a patient with a sellar lesion, with a pre-surgical diagnosis of a prolactinproducing pituitary adenoma and a finally pathological diagnosis of pituicytoma. We described clinical symptoms, signs, biochemical hormonal characteristics as well as imaging techniques used.

\section{Case details}

A 37-year-old Colombian female is admitted to the emergency department referring amenorrhoea and associated migraines from the last 3 months, she described her headaches as a pulsatile pain associate with visual and motor disturbances that had been progressively increasing and even though she consume analgesics the pain did not seem to disappear. A computerized tomography CT scan of the brain was performed showing a mass in the sellar portion of the brain compatible with an adenoma of the pituitary gland. At that time, once the headaches resolved, it was proposed surgical removal, but she refused any invasive procedure. No follow up was made until four years later when she re-consulted declaring presence of galactorrhea, and once again rejecting surgical treatment and by that time indicating treatment with cabergoline.

Symptoms progressed two years later detecting visual defects referred as diplopia and decreased visual acuity. A magnetic resonance MRI of the showed persistence of the intrasellar lesion with measurements of $12 \times 19 \times 18 \mathrm{~mm}$. with compression of the optic chiasm and extension to the right cavernous sinus with haemorrhagic areas and posterior heterogenic uptake with the administration of the dye contrast (diagnostic images, Figure 1).

Biochemical panel, showed elevated levels of prolactin $(95 \mathrm{ng} / \mathrm{mL})$ and human chorionic gonadotropin hormone. Surgical therapy was indicated and accepted by the patient. A transfenoidal approach was preferred and no complications where documented during or after de procedure. Surgical specimen description macroscopically showed yellowish and a friable consistency. Histologically, it was described as pituitary tissue with presence of malignant growth of glial origin, it also contained small and uniform astrocytes conglomerates in a bed of fibrillar tissue forming bundles. Immunohistochemical staining was reactive for fibrillar protein glial acid (FPGA), S-100, Schiff's Periodic Acid (SPA) and cytokeratin with a proliferation index of less than 1 per cent (Figure 2), do not demonstrate immunostaining for pituitary hormones (prolactin), During post-operative evaluation, central diabetes insipidus was present and 12 days later was sent home without any visual defects neither metabolic disturbances. Medical evaluations were performed at 6,13 , and 33 months after surgery without complications, any hormonal alterations neither evidence of local recurrence or metastatic disease.

\section{Discussion}

Pituicyte tumour, was first described by Bucy in 1932, is the only cell of glial origin that emerges from the posterior pituitary. (Bucy PC: The hypophysis cerebri, in Penfield W (ed): Cytology and Cellular Pathology of the Nervous System. New York, PB Hoeber, Inc., 1932, vol 2, pp705-738). It is considered that modified glial cell are positive for acidic fibrillar glial protein ${ }^{5-6}$ and showed a starred or spindleshaped along perivascular spaces linning in the infundibulum and posterior pituitary. ${ }^{7}$ In 1958, Liss and $\mathrm{Kahn}^{10}$ coined the term pituicytoma and discovered four categories of pituicytes: (a) bipolar; (b) astrocyte; (c) triangular; and (d) glomerular. In 1980, Takei described five types of pituicytes by their ultrastructural characteristics. The most common type is type (a) superior, which is similar to an astrocyte. The following represent the types of pituicytes that complete the classification: (b) dark pituicyte; (c) ependymal pituicyte; (d) oncocytic; and (e) granular. ${ }^{11}$ It is believed that each type of pituicyte can generate different types of tumours. ${ }^{4}$ Brat et al. ${ }^{7}$ described the histological characteristics of pituicytoma that are known today.

Pituicytes are not considered neurosecretory cells; however, it takes advantage of its intimate structural relationship with axonal magnocellularis to regulate hormonal releases, which explains elevated levels of prolactin and human chorionic gonadotropin in this case (stalk effect). This micro-environmental relationship is in constant changes and depends on hormonal demands. When systemic hormonal requirements are low, pituicytes take control by covering axons thus preventing their contact with basal capillary lamina and diminishing hormonal release to vascular beds. The contrary effect occurs when 
there is a physiological increase in hormonal demands. ${ }^{1,2,5,7}$ Unique histological characteristics distinguish a pituicytoma from pilocytic astrocytomas, granular cell tumours, meningiomas, and schwannomas. Pituicytomas are characterized by solid proliferation of spindle-shaped cells in fascicles interwoven. Cells are monomorphic with abundant homogeneous eosinophilic cytoplasm without granulations, oncocytic characteristics, or vacuolization. ${ }^{7}$ Characteristically, pituicytomas have a strong positive staining for S-100 protein, moderate positive stain for $\alpha 1$ anti-trypsin, $\alpha 1$ anti-chymotrypsin and Cathepsin $D$ and is focally-positive for the acidic fibrillar glial protein and antigen epithelial membrane. On the other side it does not exhibit a positive staining for synaptophysin, p53 and Schiff's Periodic Acid ${ }^{12}$ neither perinuclear reticulin and areas of Antoni A and B. ${ }^{5,13}$ Our case report exhibits the characteristics previously described.

Pilocytic astrocytoma contains microcysts, their cells are more elongated, they are highly-fibrillar and they are accompanied by Rosenthal fibers. ${ }^{7}$ Additionally, they are highly positive for the acidic fibrillar glial protein. ${ }^{1}$ Granular cell tumour consists of spindle-shaped cells even though the cytoplasm is eosinophilic with less immunoreactivity for the acidic fibrillar glial protein. ${ }^{1}$ Schwannomas contain Antoni $A$ and $B$ patterns, vascular hyalinization, perinuclear reticulate staining, and type IV collagen reactivity. ${ }^{7}$

Pituicytomas present more commonly in male gender of middle age (59 per cent). ${ }^{5}$ Clinically, it is characterized by the presence of progressive symptoms secondary to the effect of mass by the compression of neighbour structures in the brain. ${ }^{14}$ In our case, it was reported in a middle-aged woman, with migraines and symptoms related to alterations of the menstrual cycle and galactorrhea, with progression to visual symptoms. Slightly, more than one third of the patients showed visual defects specially referred and decrease in visual perimetry and acuity. Slightly more than 50 per cent of cases showed symptoms of hypopituitarism, usually manifested through sexual dysfunction, decrease in libido, and amenorrhea. ${ }^{4}$ Other, less specific symptoms are migraines, vertigo, memory changes, convulsions, and fatigue.

We only found a case report from 2006 by Benveniste et al. ${ }^{3}$ that showed a sharp increase of symptoms manifested in sudden and severe migraines without neurological deficit, with images providing evidence of a haemorrhagic suprasellar mass with drainage into the third ventricle. This shows the importance of considerations of alternative diagnoses that showed haemorrhagic suprasellar masses such as: pituicytoma, accompanied by many other pathologies such as Rathke cyst, craniopharyngioma, optical or hypothalamic glioma, cavernous haemangiomas, venous angioma, germinoma, meningioma, and aneurismatic lesions. ${ }^{3}$

Diagnostic images are unspecific, and very commonly the preoperative diagnosis is pituitary adenoma. ${ }^{15}$ Through scans, Wolfe et al. reported a tumour in the middle fossa that showed an enlargement of the Sella turcica with osseous remodelling. ${ }^{5}$ In a brain MRI, tumour are described as isointense lesions in $\mathrm{T} 1$ and hyperintense in $\mathrm{T} 2$, preformed homogeneously with the contrast medium. ${ }^{12,15}$ same pattern found in our patient.

Therapeutic options include total tumour resection through a transnasal approach which seems to give the best results and decrease local recurrence, malignant transformation or cerebrospinal manifestation. Technical difficulties related to tumour vascularization and the difficult-to-control intraoperative seemed to indicate a greater risk of relapse. ${ }^{14}$ Some authors invite an approximation by craniotomy given the tumour vascularization and its strict adherence to neighbouring structures. ${ }^{5}$ Given the apparently nonaggressive course of this type of tumour, in some cases, the subtotal resection can be the best alternative accompanied by imaging follow-up posteriorly. Equally, the decision to do adjuvant radiation and chemotherapy should be decided with regards to the aggressive biological characteristic found in this tumour, bur more studies are needed. ${ }^{14}$

\section{Conclusion}

Pituicytoma is a rare primary lesion of the sellar region. It is derived from pituicytes glial cells of the neurohypophysis or infundibulum. Pituicytoma is a low-grade spindle cell tumour (WHO grade I). In the literature are described a few cases because there has been inappropriately confused with pilocytic astrocytomas or posterior pituitary astrocytoma and infundibuloma.

\section{References}

1. Kowalski RJ, Prayson RA, Mayberg MR. Pituicytoma. Ann Diagn Pathol. 2004;8(5):290-4.

2. Ulm AJ, Yachnis AT, Brat DJ, et al. Pituicytoma: report of two cases and clues regarding histogenesis. Neurosurgery. 2004;54(3):753-7; discussion 7-8.

3. Benveniste RJ, Purohit D, Byun H. Pituicytoma presenting with spontaneous hemorrhage. Pituitary. 2006;9(1):53-8.

4. Chakraborti S, Mahadevan A, Govindan A, et al. Pituicytoma: report of three cases with review of 
literature. Pathol Res Pract. 2013;209(1):52-8.

5. Wolfe SQ, Bruce J, Morcos JJ. Pituicytoma: case report. Neurosurgery. 2008;63(1):E173-4; discussion E4.

6. Hurley TR, D'Angelo CM, Clasen RA, et al. Magnetic resonance imaging and pathological analysis of a pituicytoma: case report. Neurosurgery. 1994;35(2):314-7; discussion 7.

7. Brat DJ, Scheithauer BW, Staugaitis SM, et al. Pituicytoma: a distinctive low-grade glioma of the neurohypophysis. Am J Surg Pathol. 2000;24(3):362-8.

8. Brat DJ, Scheithauer BW, Fuller GN, et al. Newly codified glial neoplasms of the 2007 WHO classification of tumours of the central nervous system: Angiocentric glioma, pilomyxoid astrocytoma and pituicytoma. Brain Pathology. 2007;17:319-24.

9. Jenevein EP. A neurohypophyseal tumor originating from pituicytes. Am J Clin Pathol. 1964;41:522-6.

10. Liss L, Kahn EA. Pituicytoma, tumor of the sella turcica; a clinicopathological study. J Neurosurg. 1958;15(5):4818.

11. Takei Y, Seyama S, Pearl GS, et al. Ultrastructural study of the human neurohypophysis. II. Cellular elements of neural parenchyma, the pituicytes. Cell Tissue Res. 1980;205(2):273-87.

12. Kosuge $\mathrm{Y}$, Hiramoto J, Morishima $\mathrm{H}$, et al. Neuroimaging characteristics and growth pattern on magnetic resonance imaging in a 52-year-old man presenting with pituicytoma: a case report. J Med Case Rep. 2012;6(1):306.

13. Nakasu Y, Nakasu S, Saito A, et al. Pituicytoma. Two case reports. Neurol Med Chir (Tokyo). 2006;46(3):152-6.

14. Pirayesh Islamian A, Buslei R, Saeger $W$, et al. Pituicytoma: overview of treatment strategies and outcome. Pituitary. 2012;15(2):227-36.

15. Secci F, Merciadri P, Rossi DC, et al. Pituicytomas: radiological findings, clinical behavior and surgical management. Acta Neurochir (Wien). 2012;154(4):64957; discussion 57.

\section{ACKNOWLEDGEMENTS}

Fernando Velandia Hurtado for your dedication in the clinical practice

\section{PEER REVIEW}

Not commissioned. Externally peer reviewed.

\section{CONFLICTS OF INTEREST}

The authors declare that they have no competing interests.

\section{FUNDING}

None

\section{PATIENT CONSENT}

The authors, Velandia Hurtado F, Aponte JE, Madriñan $H$, Forero $Y$, Anturi YC, declare that:

1. They have obtained written, informed consent for the publication of the details relating to the patient(s) in this report.

2. All possible steps have been taken to safeguard the identity of the patient(s).

3. This submission is compliant with the requirements of local research ethics committees.

Figure 1: T1W1 with contrast dye, Coronal and Sagittal Cerebral magnetic resonance images
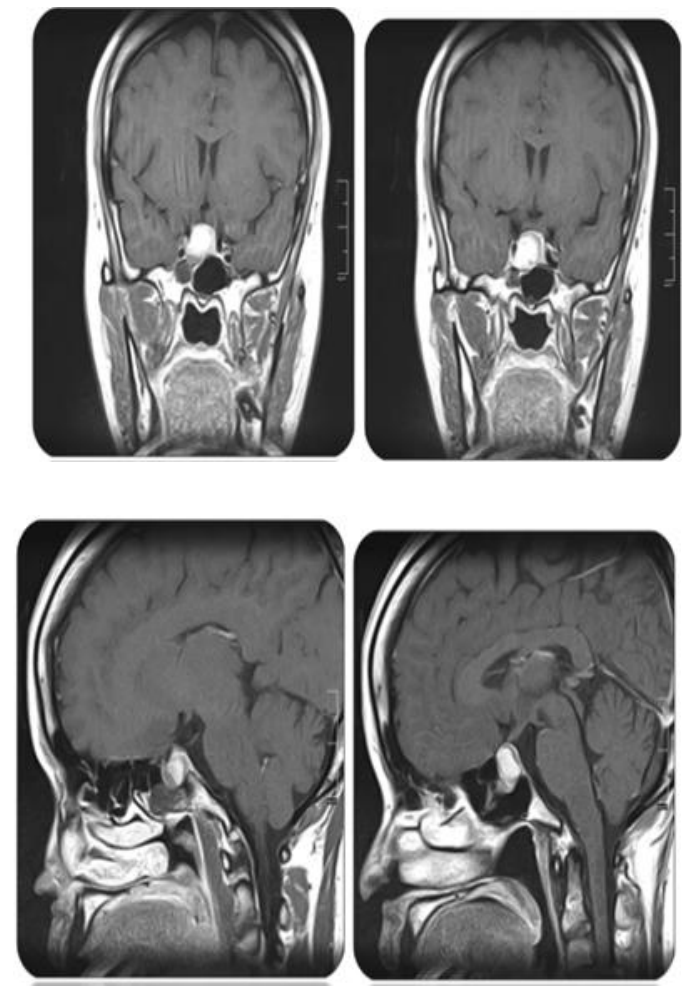
Figure 2: Images of histhopatological study
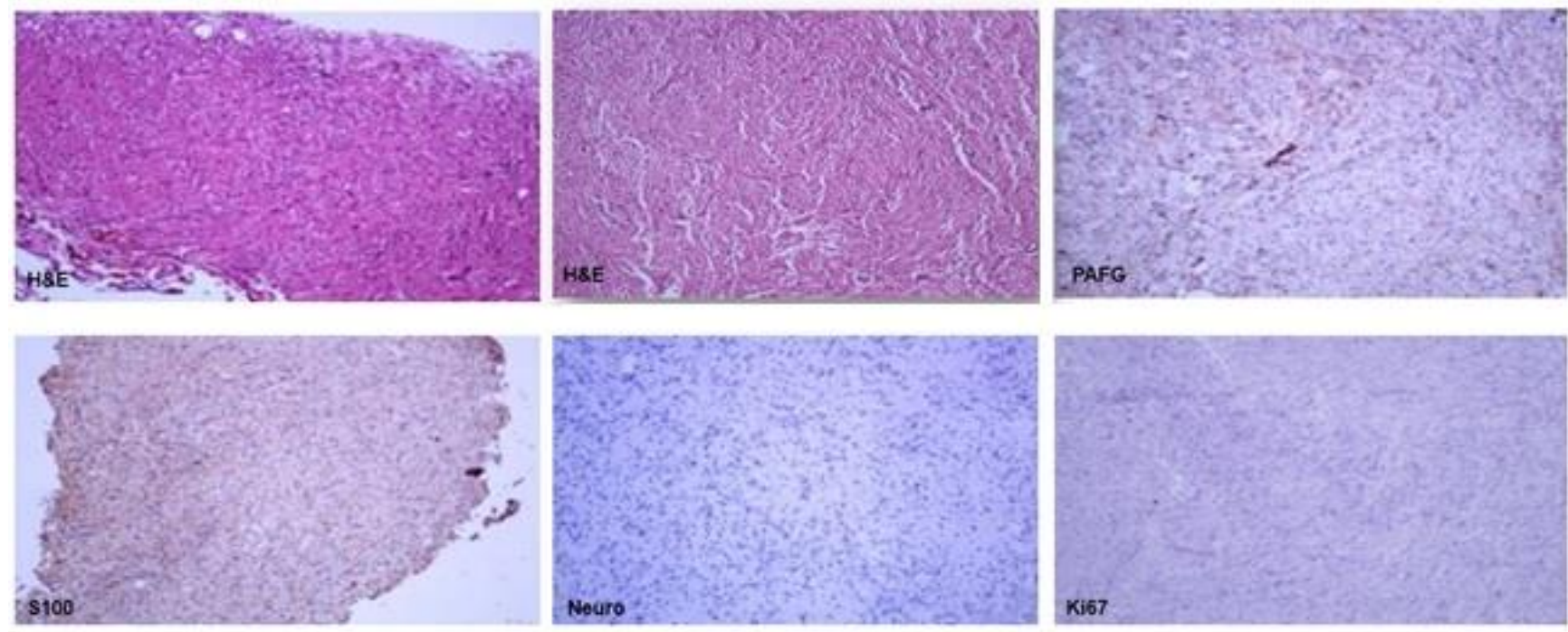